\title{
Observations of the effect of strong Pauli paramagnetism on the vortex lattice in superconducting $\mathrm{CeCu}_{2} \mathrm{Si}_{2}$
}

\author{
E. Campillo $\odot,{ }^{1,2, *}$ R. Riyat, ${ }^{2}$ S. Pollard $\odot,{ }^{2}$ P. Jefferies, ${ }^{2}$ A. T. Holmes $\odot,{ }^{3}$ R. Cubitt, ${ }^{4}$ J. S. White $\odot,{ }^{5}$ J. Gavilano, ${ }^{5}$ \\ Z. Huesges,${ }^{6,7}$ O. Stockert, ${ }^{6}$ E. M. Forgan, ${ }^{2}$ and E. Blackburn ${ }^{1,2, \dagger}$ \\ ${ }^{1}$ Division of Synchrotron Radiation Research, Lund University, SE-22100 Lund, Sweden \\ ${ }^{2}$ School of Physics and Astronomy, University of Birmingham, Birmingham B15 2TT, United Kingdom \\ ${ }^{3}$ European Spallation Source ERIC, P.O. Box 176, SE-221 00, Lund, Sweden \\ ${ }^{4}$ Institut Laue Langevin, 71 Avenue des Martyrs, F-38042 Grenoble Cedex 9, France \\ ${ }^{5}$ Laboratory for Neutron Scattering and Imaging (LNS), Paul Scherrer Institute (PSI), CH-5232 Villigen, Switzerland \\ ${ }^{6}$ Max Planck Institute for Chemical Physics of Solids, DE-01187 Dresden, Germany \\ ${ }^{7}$ Helmholtz-Zentrum Berlin für Materialien und Energie GmbH, 14109 Berlin, Germany
}

(Received 10 June 2021; revised 19 October 2021; accepted 26 October 2021; published 12 November 2021)

\begin{abstract}
We present the results of a study of the vortex lattice in the heavy fermion superconductor $\mathrm{CeCu}_{2} \mathrm{Si}_{2}$, using small-angle neutron scattering (SANS). In this material at temperatures well below $T_{\mathrm{c}} \sim 0.6 \mathrm{~K}$, the value of the upper critical field $B_{\mathrm{c} 2} \sim 2.2 \mathrm{~T}$ is strongly limited by the Pauli paramagnetism of the heavy fermions. In this temperature region, our SANS data show an increase in the magnetization of the flux line cores with field, followed by a rapid fall near $B_{\mathrm{c} 2}$. This behavior is the effect of Pauli paramagnetism and we present a theory-based model, which can be used to describe this effect in a range of materials. The pairing in $\mathrm{CeCu}_{2} \mathrm{Si}_{2}$ appears to arise from the effect of magnetic fluctuations, but the evidence for a $d$-wave order parameter is rather weak. We find that the vortex lattice structure in $\mathrm{CeCu}_{2} \mathrm{Si}_{2}$ is close to regular hexagonal. There are no phase transitions to square or rhombic structures; such transitions are expected for $d$-wave superconductors and observed in $\mathrm{CeCoIn}_{5}$; however, the temperature dependence of the SANS intensity indicates that both large and small gap values are present, most likely due to multiband $s$-wave superconductivity, rather than a nodal gap structure.
\end{abstract}

DOI: 10.1103/PhysRevB.104.184508

\section{INTRODUCTION}

$\mathrm{CeCu}_{2} \mathrm{Si}_{2}$ was the first example of a heavy fermion superconductor [1], where the many-body interactions strongly renormalize the electronic properties. The low-temperature physical properties are very sensitive to extremely small changes in the stoichiometry [2]. This means that with minor variations in the $\mathrm{Cu}$ content, samples that are antiferromagnetic (A-type), superconducting (S-type), or both (A/S-type) can be prepared. A stoichiometric $\mathrm{CeCu}_{2} \mathrm{Si}_{2}$ sample exhibits both antiferromagnetic and superconducting properties (A/Stype); a slight excess of copper gives an S-type sample.

Since the discovery of $\mathrm{CeCu}_{2} \mathrm{Si}_{2}$, it was clear that the upper critical field $(\sim 2 \mathrm{~T})$ for the superconducting state is lower than expected. This is usually an indication that the superconductivity is not destroyed by the typical orbital mechanism, but instead by Pauli paramagnetic effects (PPE). The

*emma.campillo@sljus.lu.se

†elizabeth.blackburn@sljus.lu.se

Published by the American Physical Society under the terms of the Creative Commons Attribution 4.0 International license. Further distribution of this work must maintain attribution to the author(s) and the published article's title, journal citation, and DOI. Funded by Bibsam.
Pauli spin susceptibility acts to align unpaired spins parallel to the applied magnetic field; this competes with the expected antiparallel spin alignment for a Cooper pair in a singlet superconductor [3]. If the contribution from the Pauli spin susceptibility is large enough, this gives a low-temperature paramagnetic limiting field $B_{\mathrm{P}}=\Delta / \sqrt{ } 2 \mu_{B}$, which is below the value $B_{\mathrm{c} 2}^{\text {orb }}$ expected from the orbital mechanism. The relative strength of the two mechanisms is described by the Maki parameter, $\alpha_{\mathrm{M}}=\sqrt{ } 2 B_{\mathrm{c} 2}^{\text {orb }} / B_{\mathrm{P}}$ [4]. For conventional superconductors, this has a value lower than 1 ; for $\mathrm{CeCu}_{2} \mathrm{Si}_{2}$, $\alpha_{\mathrm{M}}=9.5$ [5] and the observed low-temperature value of $B_{\mathrm{c} 2}$ is limited to a value $\sim B_{\mathrm{P}}$. Strong PPE are also expected to favour the formation of a Fulde-Ferrell-Larkin-Ovchinnikov (FFLO) spatially modulated superconducting state [6,7]. Direct proof for this state remains elusive, but Kitagawa et al. [5] report a proposed signature in their nuclear magnetic resonance measurements in the high-field and low-temperature part of the phase diagram.

Extensive transport and thermodynamic studies have been undertaken on this compound, to study both the antiferromagnetic and the superconducting order parameters [8]. Inelastic neutron scattering results relate the superconducting pairing energy to spin excitations, rather than phonons [2]. Angle-resolved resistivity measurements of $B_{\mathrm{c} 2}$ show a fourfold oscillation in the critical field, suggesting a $d_{x y}$ symmetry of the order parameter [9]. However, specific-heat measure- 


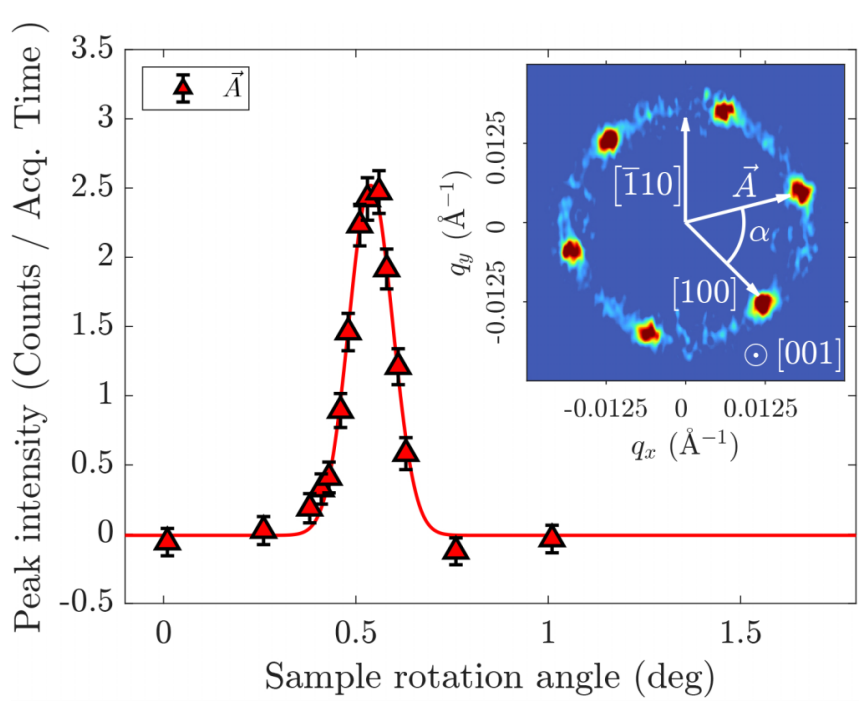

FIG. 1. Rocking curve in $\omega$ at $130 \mathrm{mK}$ and $1.5 \mathrm{~T}$ for the reflection labeled $\vec{A}$ in the inset, which shows the diffraction pattern of the vortex lattice in $\mathrm{CeCu}_{2} \mathrm{Si}_{2}$ obtained by summing $\omega$ and $\phi$ rocking scans with the field applied along $\mathbf{c}$. One pair of spots is aligned with the crystal [100] direction.

ments point to the existence of two nodeless superconducting gaps $[10,11]$. Several different theoretical models for the gap structure have been put forward $[12,13]$.

In this paper, we study the magnetic vortex lattice (VL) in an S-type sample using small-angle neutron scattering (SANS). In most superconductors, the neutron scattering intensity from the vortex lattice decreases exponentially with applied magnetic field, as the vortex cores come closer together. In $\mathrm{CeCoIn}_{5}$, the observed intensity increases towards $B_{\mathrm{c} 2}$, before dropping rapidly just before $B_{\mathrm{c} 2}$ [14]. This anomalous behavior is ascribed to PPE, which increase the relative strength of the magnetization in the vortex cores. This has been modelled by Ichioka and Machida [15] using selfconsistent microscopic calculations based on quasiclassical Eilenberger theory. Much weaker anomalies are reported in some other superconductors $\left(\mathrm{TmNi}_{2} \mathrm{~B}_{2} \mathrm{C}\right.$ [16], $\mathrm{YBa}_{2} \mathrm{Cu}_{3} \mathrm{O}_{7}$ [17]), and are thought to have the same origin. We now report that $\mathrm{CeCu}_{2} \mathrm{Si}_{2}$ shows similar behavior to $\mathrm{CeCoIn}$, and present a calculation-based model that captures the behavior in materials exhibiting PPE, without requiring numerically intensive calculation.

\section{VORTEX LATTICE ORIENTATION AND DISTORTION}

In Fig. 1, there are six spots, with the strongest spots lying along a [100]-type direction. $\mathrm{CeCu}_{2} \mathrm{Si}_{2}$ is tetragonal, so when the magnetic field is parallel to $\mathbf{c}$, two degenerate $\mathrm{VL}$ domain orientations should be observed, at $90^{\circ}$ to each other. However, in this image, it is clear that one domain is preferentially favored; we have observed this in all of our experiments. The second domain does exist, but is significantly weaker. We do not have clear evidence as to why this arises; we postulate that there is some small anisotropy between the [100] and [010] directions, perhaps due to a small misalignment of the sample with respect to the field, or related to the sample growth. (a)

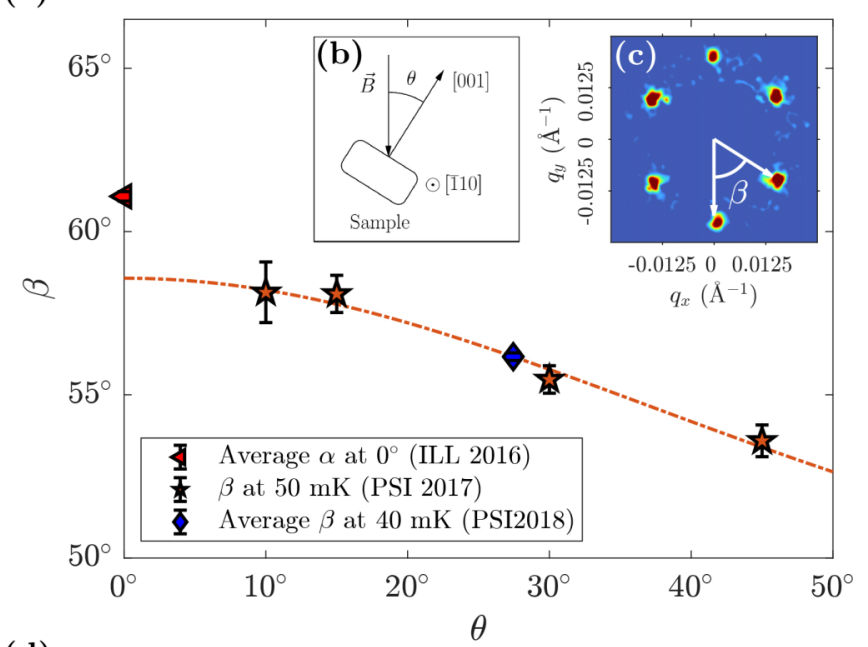

(d)

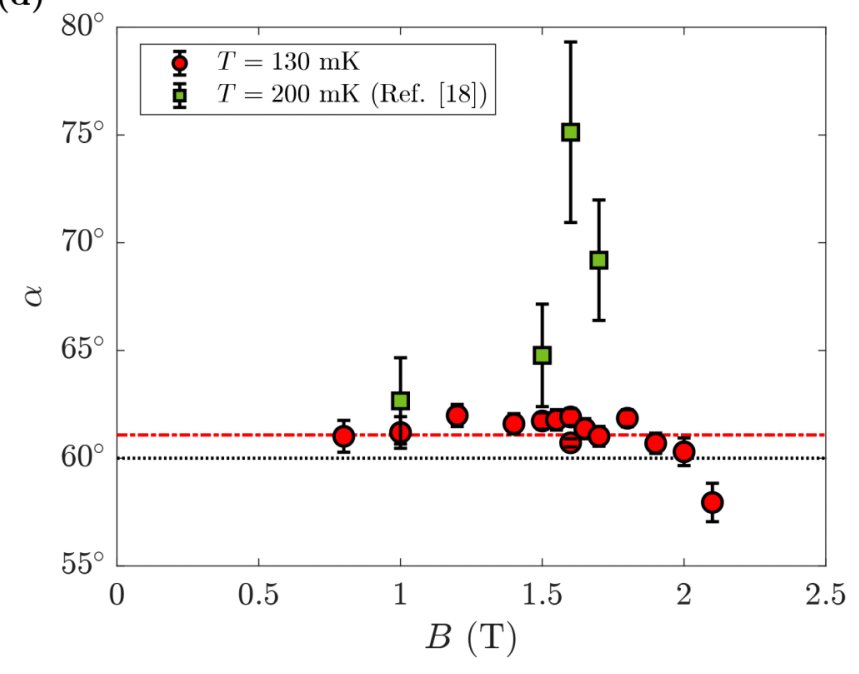

FIG. 2. (a) $\theta$ angle dependence of the opening angle $\beta$ between the bottom diffraction spot, which lies on the [1 $\overline{1} 0]$ direction, and the bottom right spot. The data were obtained at $1.5 \mathrm{~T}$ and base temperature. The curve is a fit to Eq. (9). The $\diamond$ point corresponds to the average of all $\beta$ angles measured at $40 \mathrm{mK}$. The $\triangleleft$ point at $\theta=0^{\circ}$ is the average of all $\alpha$ angles measured at $130 \mathrm{mK}$ [Fig. 5(d)], and is not included in the fit as it corresponds to a different VL orientation. (b) Schematic of the setup where $\theta$ is the angle between the incoming magnetic field and the $\mathbf{c}$ axis of the sample. (c) Diffraction pattern of the vortex lattice in $\mathrm{CeCu}_{2} \mathrm{Si}_{2}$ obtained at $\theta=30^{\circ}, 50 \mathrm{mK}$ and $1.5 \mathrm{~T}$. (d) Field dependence of the opening angle $\alpha$ between the directions [100] and $\vec{A}$ at $130 \mathrm{mK}$. The green squares represent the anisotropy measured in previous STM experiments [18].

In Fig. 2 we show measurements at $50 \mathrm{mK}$ and $1.5 \mathrm{~T}$ of the changes in the vortex lattice on rotating the $\mathbf{c}$ axis of the sample with respect to the magnetic field by an angle $\theta$ about the vertical axis [Fig. 2(b)]. Above $\theta=5^{\circ}$, the vortex lattice changes its orientation from that in Fig. 1 to that in Fig. 2(c), with two spots along the vertical [110]-type direction. With this sample rotation, the vertical [110]-type direction remains unchanged, but is distinguished from the horizontal direction, which is now of type $[H H L]$. The reorientation of the VL indicates that there is no strong locking of the vortex lattice to the underlying crystal structure, and $a c$ anisotropy plus 
rotation can drive the vortex lattice to a new orientation. This indirectly supports the hypothesis that the superconducting gap is not nodal, and is likely to be $s$ wave.

At all tilt angles, the lattice is not quite a perfect hexagonal shape, but instead a hexagon circumscribed by an ellipse. We have characterized this distortion by measuring the angle $\beta$ between the bottom and bottom-right spots for $10^{\circ} \leqslant \theta \leqslant$ $45^{\circ}$. Supposing that there is an effective mass anisotropy $\Gamma$ between the directions $\perp$ and $\|$ to the $\mathbf{c}$ axis, the data are fitted to the expression [19]:

$$
\tan \beta=\left[\frac{3}{\Gamma_{0}\left(\cos ^{2} \theta+\Gamma \sin ^{2} \theta\right)}\right]^{\frac{1}{2}}
$$

Here, $\sqrt{ } \Gamma_{0}=1.06(2)$ allows for distortion of the hexagonal lattice at $\theta=0$ by the fourfold crystal symmetry [20]. From fitting the $\theta$ dependence we obtain $\sqrt{ } \Gamma=1.40(7)$. In conventional superconductors, the anisotropy of the upper critical field is given by $B_{c 2, c} / B_{c 2, a b}=\sqrt{ } \Gamma$ [21]. Values of upper critical fields obtained from heat capacity measurements [11] yield $\sqrt{ } \Gamma=1.14$ at low temperatures. However, these critical fields are strongly Pauli limited and do not directly reflect the anisotropy of coherence length. It appears more appropriate to use the estimates for the orbitally-limited critical fields reported in the same publication, which give $\sqrt{ } \Gamma=1.47$, close to our result from VL distortion.

In Fig. 2(d), the angle $\alpha$ (defined in Fig. 1) measured at $130 \mathrm{mK}$ shows little change with field, being close to regular hexagonal, with a a small change in anisotropy at high field. In a previous STM study, a big distortion was reported at $1.6 \mathrm{~T}$ [18]; however, we observe only a slight distortion of the lattice near this field and nothing of the magnitude and abruptness reported in the STM paper, perhaps because we are observing the bulk, not the surface of the sample. The small VL distortions we observe at high field may be linked to PPE or even the proposed onset of the FFLO state [5], but are so small that they rule out $d$-wave effects.

\section{PAULI PARAMAGNETIC EFFECTS ON THE MAGNETIC FIELD DISTRIBUTION}

The magnetic field in the VL may be expressed as a sum of Fourier components in $\mathbf{q}$ space,

$$
B(\mathbf{r})=\sum_{h k} F\left(\mathbf{q}_{h k}\right) e^{i \mathbf{q}_{h k} \cdot \mathbf{r}} .
$$

The magnitude of each Fourier component $\left|F\left(\mathbf{q}_{h k}\right)\right|$, often called the "form factor", is extracted from the measured integrated intensity $I\left(\mathbf{q}_{h k}\right)$ of the vortex lattice Bragg peaks using the Christen formula [23]:

$$
I\left(\mathbf{q}_{h k}\right)=2 \pi V \phi_{n}\left(\frac{\gamma}{4}\right)^{2} \frac{\lambda_{n}^{2}}{\Phi_{0}^{2} q_{h k} \cos (\zeta)}\left|F\left(\mathbf{q}_{h k}\right)\right|^{2},
$$

where $V$ is the sample volume, $\phi_{n}$ is the flux of neutrons of wavelength $\lambda_{n}, \gamma$ is the magnetic moment of a neutron, $\Phi_{0}$ is the flux quantum, $q_{h k}$ is the magnitude of the scattering vector, and $\cos (\zeta)$ is the Lorentz correction, where $\zeta$ is the angle between the reciprocal lattice vector $\mathbf{q}$ and the direction perpendicular to the sample rotation axis.
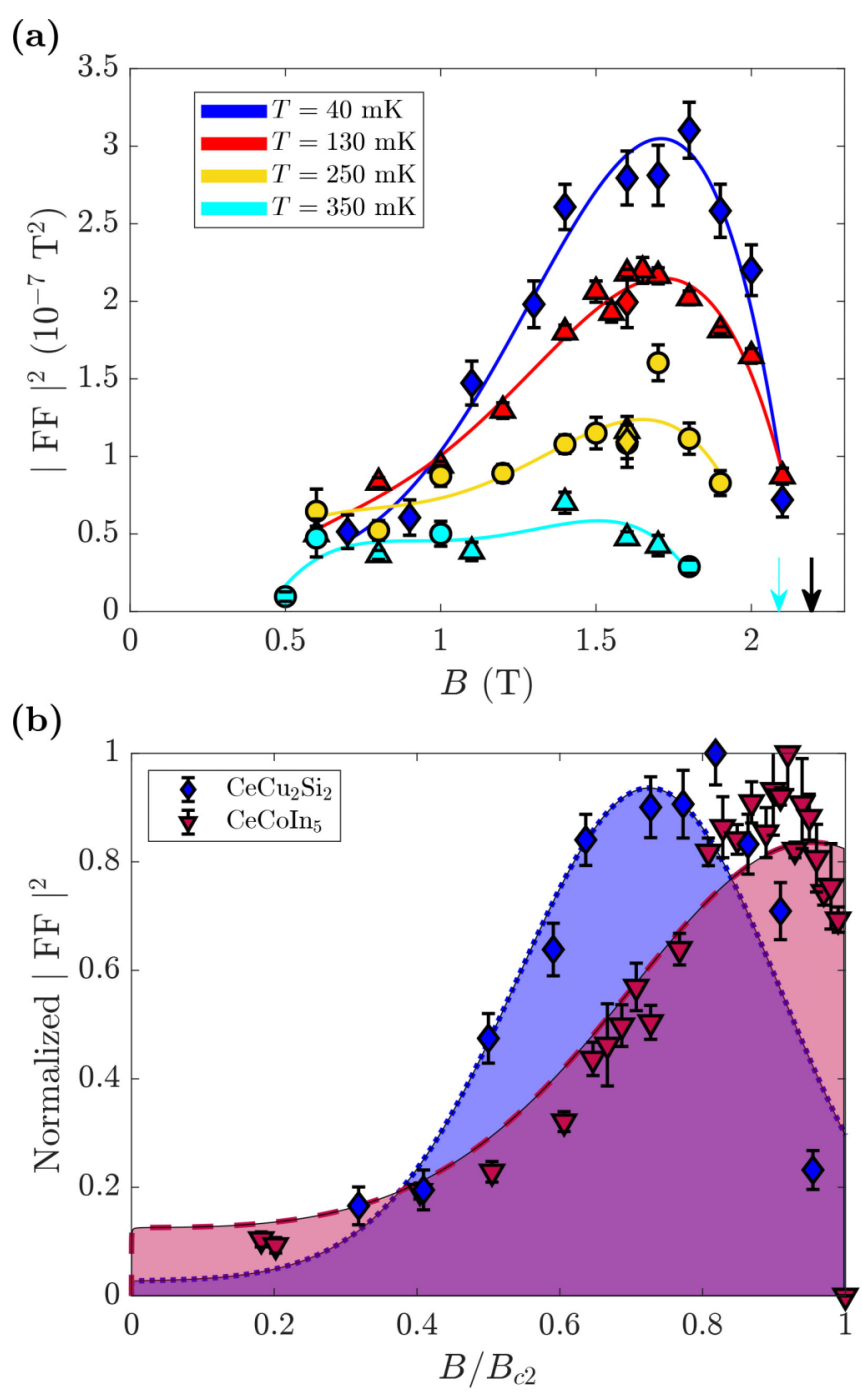

FIG. 3. (a) Field dependence of the squared form factor calculated from the first order diffraction spots with a well-defined rocking curve, for temperatures between $40 \mathrm{mK}$ and $350 \mathrm{mK}$. The symbol $(\triangle)$ corresponds to the data obtained at ILL in 2016, ( $)$ corresponds to data obtained at ILL in 2018, and $(\diamond)$ data were obtained at PSI in 2018. $\diamond$ data were measured with the sample rotated away from the $\mathbf{B} \| \mathbf{c}$ orientation [see Fig. 2(a)] but the integrated intensity is not affected by this change, as we can observe from the points at $1.6 \mathrm{~T}$, $250 \mathrm{mK}$ and $130 \mathrm{mK}$, that match with measurements from other experiments. The cyan arrow corresponds to the value of $B_{\mathrm{c} 2}(T)$ for $T=350 \mathrm{mK}$ and the wide black arrow covers all the upper critical fields for $T=40,130$, and $250 \mathrm{mK}$ obtained from Ref. [5]. Solid lines are guides for the eye and the crossing of the lines is not real [simulated dependence at low fields shown in Fig. 4(a)]. (b) Comparison between the normalized squared form factor of $\mathrm{CeCu}_{2} \mathrm{Si}_{2}$ at $40 \mathrm{mK}$ and $\mathrm{CeCoIn}_{5}$ at $50 \mathrm{mK}$ [22]. The lines are the results of fits using a model for PPE described in the next section.

In Fig. 3(a), we show the field dependence of the form factor of the first order reflections from the VL at $T=40,130,250$, and $350 \mathrm{mK}$. For these measurements, $B \|$ $c$ except at $40 \mathrm{mK}$, where there is an offset of 28 degrees [see Fig. 2(a)]. As shown in Fig. 3(a) this does not appear to affect the observed intensities. The form of the field-dependence 
changes with temperature, with PPE visible over almost the entire field range. In general, the form factor rises up to a maximum, and then begins to fall to zero on approaching $B_{\mathrm{c} 2}$, indicating strong PPE. For $40 \mathrm{mK}$ and $130 \mathrm{mK}$ the intensity falls steeply on reaching $\sim 2.1 \mathrm{~T}$, showing a rapid variation on approaching the normal state. At $250 \mathrm{mK}$ the form factor increases slightly with field, showing that PPE remain important but we do not observe a sudden decrease near $B_{\mathrm{c} 2}$. Meanwhile, at $350 \mathrm{mK}$ the form factor is more constant as a function of field, but falling towards zero at low and high fields.

This kind of behavior is also observed in $\mathrm{CeCoIn}_{5}$ [22] [see Fig. 3(b)]. However in this material, a first order transition into the normal state at $B_{\mathrm{c} 2}$ is clearly observed at low temperatures, both in heat capacity and SANS. This is in contrast to the second-order transition observed in $\mathrm{CeCu}_{2} \mathrm{Si}_{2}$, again both in heat capacity [10] and in our SANS data.

In both materials, the field-dependence cannot be described by the various standard models for the form factor associated with vortex lattices. A brief discussion of such models is therefore given in the Supplemental Material [24].

\section{MODELLING OF VORTEX CORE MAGNETIZATION AND PPE}

To better understand the behavior of $\mathrm{CeCu}_{2} \mathrm{Si}_{2}$ in the mixed state, we have derived a physically-based algebraic expression for the form factor in Pauli-limited superconductors, based on the results of first-principles computation [15,25]. This shows that Pauli-limiting leads to two effects: (i) additional spin magnetization developing in the cores, and (ii) the core radius growing with increasing field. One effect of the finite size of the cores is that they will tend to suppress the form factor at large $q$. This will apply both for the paramagnetic contribution $F_{\mathrm{PM}}$ and also the small contribution from orbital currents $F_{\text {orb }}$ described in the Supplemental Material [24]. We represent this by the Brandt model [26], with a Gaussian cut-off depending on a now field-dependent coherence length $\xi_{\mathrm{PM}}$ and the value of $q$ :

$$
F(B)=\exp \left(-c q^{2} \xi_{\mathrm{PM}}^{2}\right)\left(F_{\mathrm{orb}}(B)+F_{\mathrm{PM}}(B)\right),
$$

where the constant $c$ is a parameter expected to have a value between 0.25 and 0.5 . A value for $c$ of 0.42 was found in $\mathrm{YBa}_{2} \mathrm{Cu}_{3} \mathrm{O}_{7}$ [27], and $q^{2}$ is proportional to $B$ by flux quantization.

For the expansion of the core size by paramagnetic effects, we take $\xi_{\mathrm{PM}}$ to vary quadratically with $B$ at low fields and linearly at high fields [25]:

$$
\xi_{\mathrm{PM}}^{2}(B, T)=\xi_{\mathrm{orb}}^{2}(T)+\epsilon\left(\frac{\Phi_{0}}{2 \pi B_{\mathrm{c} 2}(T)}\right)\left(\frac{B}{B_{\mathrm{c} 2}(T)}\right)^{2},
$$

where $\xi_{\text {orb }}$ is the core size in the absence of PPE and $B_{\mathrm{c} 2}(T)$ is the experimental upper critical field at temperature $T$. The $B_{\mathrm{c} 2}(T)$ factors have been included so that the fitting parameter $\epsilon$ will be $\sim 1$ and fairly temperature independent if $\xi_{\mathrm{PM}}$ is comparable with the vortex spacing at $B=B_{\mathrm{c} 2}(T) . \epsilon$ can be interpreted as a correction of the size of the core when PPE are present.

For the value and temperature dependence of the core size $\xi_{\text {orb }}$, we have to consider several effects. One length scale is the BCS coherence length $\xi_{\mathrm{BCS}}(T)=\hbar v_{F} / \pi \Delta(T)$.
As Kramer and Pesch pointed out [28], in clean type-II materials the core contracts strongly at low temperatures:

$$
\xi_{\text {orb }}(T) \sim \xi_{\mathrm{BCS}}(T) \times T / T_{c}
$$

This is because $\Delta(r)$ is self-consistently determined by the wave functions in the core of the occupied unpaired states, and these are restricted to smaller $r$ as the temperature is lowered. If the material is not perfectly clean, the core shrinkage stops below a temperature given by $T / T_{c}<\xi_{\mathrm{BCS}} / \ell$. As shown in the Supplemental Material [24], this ratio is $\sim 0.25$. We shall therefore use Eq. (6) to give the temperature dependence of $\xi_{\text {orb }}(T)$ down to $T=0.25 T_{c}$, with a constant multiplier below that temperature. We take $\xi_{\mathrm{BCS}}(T=0)=$ $\Phi_{0} / 2 \pi B_{\mathrm{c} 2}^{\mathrm{orb}}(T=0)$, with the orbital-limiting field at zero temperature given by $B_{\mathrm{c} 2}^{\mathrm{orb}}(T)=B_{\mathrm{c} 2}^{\mathrm{orb}}(0) \sum_{i} w_{i}\left(\Delta_{i}(T) / \Delta_{i}(0)\right)^{2}$, assuming $\xi_{\text {orb }}(T) \propto 1 / \Delta(T)$ and where $\Delta_{i}(T)$ are the different superconducting gaps present and $w_{i}$ are their respective weights. At low field values, the increase in core size with temperature will tend to increase the paramagnetic contribution to the form factor; however, at high fields the form factor will drop due to the effect of the Gaussian cut off.

At $T=0$, we expect the spin susceptibility outside the cores to be zero so there will be a full magnetic contrast between vortex cores and the remainder of the VL. Hence the paramagnetic form factor will be proportional to the core magnetization. This rises rapidly, proportional to magnetic field up to $\sim 0.1 B_{\mathrm{c} 2}$, but in the field range where we have measured, it varies little with applied field [25] with a value $\mu_{0} M \sim \chi_{n} \mu_{0} H_{c 2} \sim \chi_{n} B_{\mathrm{c} 2}$, where $\chi_{n}$ is the normal state Pauli susceptibility at $T=0$. With increasing temperature, the quasiparticles excited above $\Delta$ will increase $M$ outside the cores and decrease the contrast, while $\chi_{n}$ will remain approximately constant. Assuming that $\Delta$ between the cores is not strongly suppressed by field, the spin susceptibility in this region is given by [29]

$$
\frac{\chi_{s}(T)}{\chi_{n}}=\sum_{i} 2 w_{i} \int_{0}^{\infty} d y \frac{\exp \left[\left(y^{2}+\beta^{2} \Delta_{i}^{2}(T)\right)^{\frac{1}{2}}\right]}{\left\{\exp \left[\left(y^{2}+\beta^{2} \Delta_{i}^{2}(T)\right)^{\frac{1}{2}}\right]+1\right\}^{2}}
$$

where $\beta=1 / k_{B} T$ and $w_{i}$ is the weight of a band (if multiband) having energy gap $\Delta_{i}(T)$. Hence the magnetization contrast $\mu_{0} \delta M$ in the VL becomes $\sim\left[\chi_{n}-\chi_{s}(T)\right] B_{\mathrm{c} 2}$. A calculation of the fraction of normal fluid in the superconducting state [30] gives the same integral as Eq. (7). Hence we can write the contrast in terms of the superfluid fraction $\rho_{s}(T) / \rho$ to give $\mu_{0} \delta M \sim \chi_{n} B_{\mathrm{c} 2} \times \rho_{s}(T) / \rho$. To calculate $\rho_{s}(T)$, we need the temperature-dependence of the superconducting gap function, which is taken as [30]

$$
\Delta_{i}(T)=\Delta_{i}(0) \tanh \left(\frac{\pi k_{B} T_{c}}{\Delta_{i}(0)} \sqrt{a\left(\frac{T_{c}}{T}-1\right)}\right),
$$

where $\Delta_{i}(0)$ is the value of the gap at zero temperature. Some quantities in this equation depend on the pairing state of the superconductor: in the case of BCS isotropic $s$ wave, $\Delta(0)=$ $1.76 k_{B} T_{c}$ and $a=1$.

The Pauli paramagnetic contribution to the form factor depends on the magnetization contrast, the area of a flux line and the number of flux lines per unit area. Hence, apart from 
(a)

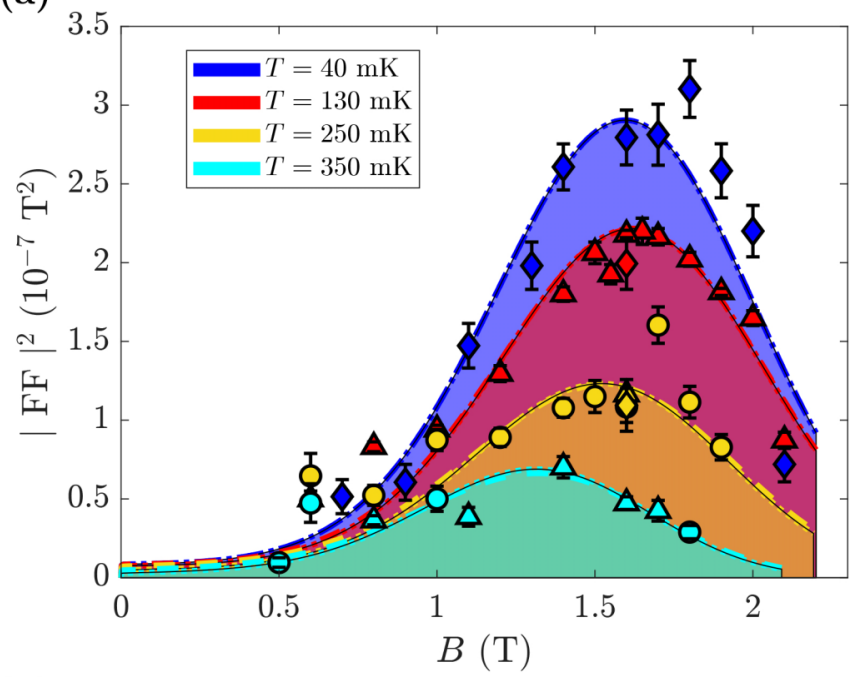

(b)

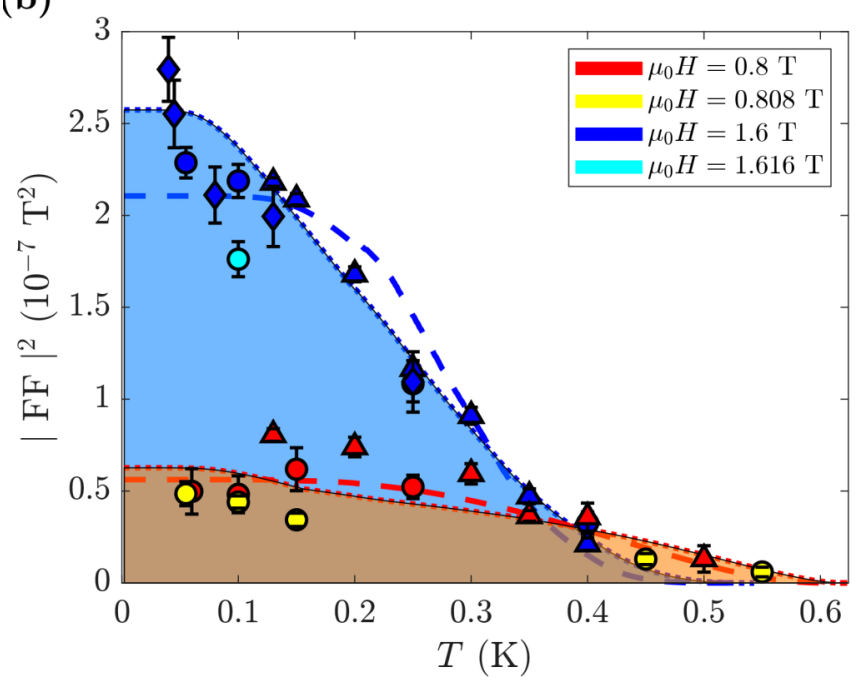

FIG. 4. (a) Field and (b) temperature dependence of the squared form factor with the results of the fits using our model for paramagnetic effects, with a single $s$-wave gap (dashed line) and two-gap $s$-wave model (dotted line). For all fits $\lambda(0)=7000 \AA$ [33].

the Gaussian core correction factor, we have

$$
F_{\mathrm{PM}}(B, T)=\eta\left(\frac{2 \pi B}{\Phi_{0}}\right)\left[\left(\frac{\rho_{s}(T)}{\rho}\right) \chi_{n} B_{\mathrm{c} 2}(T)\right] \xi_{\mathrm{PM}}^{2}(B, T),
$$

where the constant $\eta$ is a fitting parameter expected to have a value of $\sim 1$ included for correcting any approximations made for the magnetization in the core.

On the other hand, the orbital contribution to the form factor [Eq. (S1), Ref. [24]] has a temperature dependence from the London penetration depth, which can also be derived from the superfluid fraction, $\rho_{s}(T) / \rho=(\lambda(0) / \lambda(T))^{2}$. The orbital and the paramagnetic contributions [Eq. (9)] can then be substituted into Eq. (4) to give an algebraic model to fit our data as a function of field and temperature. Figure 4(a) shows the fits versus field at various fixed temperatures, using a single $s$-wave and a two-band $s$-wave model, with the latter using the gap values and weights proposed in previous specific heat measurements [10]. In Table I are given the fitting parameters and the root-mean-square deviation between the curves and the data. Both models capture the behavior up to the peak quite well, but are unable to reproduce the sharp drop on the approach to $B_{\mathrm{c} 2}$ at low temperatures. This could be due to a suppression of $\Delta$ between the cores at high fields and low temperatures, or it could be an effect of the FFLO state proposed by Kitagawa et al. [5], as the FFLO state is expected to suppress the form factor [31,32]. The fitting parameters $\epsilon$ and $\eta$ give sufficient flexibility that the two models fit equally well.

\section{TEMPERATURE DEPENDENCE OF THE INTENSITY OF VORTEX LATTICE SIGNAL}

In Fig. 4(b) we plot the temperature dependence of the form factor of the first order reflections from the VL at $B=$ $0.8 \mathrm{~T}$ (combined with points at $0.808 \mathrm{~T}$ ) and $1.6 \mathrm{~T}$ (combined with points at $1.616 \mathrm{~T}$ ). Figure 4(b) also shows the fits using the $s$-wave model at $0.8 \mathrm{~T}$ and $1.6 \mathrm{~T}$, and the $s+s$-wave model again using the values of the gaps and weights proposed by Kittaka et al. [10]. The values of the fit parameters are in Table I, including the root-mean-square deviation for each fit. A two-gap model is required to fit heat capacity data in this material $[10,11]$. For our SANS data, we find that the two-gap model gives parameters that are less temperature dependent and have better correspondence between field and temperature scans. The rise in the $1.6 \mathrm{~T}$ form factor at low temperatures seen in Fig. 4(b) cannot be reproduced by a single-gap model, so we prefer the two-gap model, although our fits do not enforce it.

\section{DISCUSSION}

In this paper we have measured the field-dependence of the form factor of the vortex lattice in $\mathrm{CeCu}_{2} \mathrm{Si}_{2}$, giving a measure of the magnetization of the unpaired electrons inside the cores, which is particularly important at high field and low temperatures. The field- and temperature dependence of our results sheds light on the effects of Pauli paramagnetism on vortex core size and which gap model works best. The extent of our investigations, and the underlying physics involved is summarised in Fig. 5.

Our measurements of the field-dependence of the form factor represent the second observation of Pauli paramagnetic effects in a heavy-fermion material, after the observations made in $\mathrm{CeCoIn}_{5}$ [14,22]. Unlike $\mathrm{CeCoIn}_{5}, \mathrm{CeCu}_{2} \mathrm{Si}_{2}$ does not show a first-order transition to the normal state at high fields. Both materials have some evidence supporting the existence of a field-induced quantum critical point [34]. We note that rather weaker effects due to the paramagnetism of localised $f$ electrons have also been observed in $\mathrm{TmNi}_{2} \mathrm{~B}_{2} \mathrm{C}$ [16].

Near $B_{\mathrm{c} 2}$ at low temperatures the observed intensity drops faster than expected. One possible explanation for this could be that the material enters the postulated FFLO phase, which would suppress the form factor (see Fig. $3 \mathrm{~b}$ in Ref. [31] and Fig. 5 in Ref. [32]). This observation also matches with previous NMR studies [5] in which an enhancement of $1 / T_{1} T$, observed just below the upper critical field is taken as evidence for the FFLO state (see Fig. 5). We see in Fig. 4(a) that the maximum peak intensity is shifted towards the upper critical field for decreasing temperature, suggesting a major contribu- 
TABLE I. Parameters resulting from the fits and the root-mean-square deviations for field scans in Fig. 4(a) and temperature scans in Fig. 4(b). For the $s$-wave fit, we used the value $\Delta(0)=1.76 k_{B} T_{c}$, and for the $s+s$ wave the two values of the superconducting gaps were $\Delta_{1}(0)=1.76 k_{B} T_{c}$ and $\Delta_{2}(0)=0.7 k_{B} T_{c}$, with weights $w_{1}=0.65$ and $w_{2}=0.35$ as given by the heat capacity data in [10]. In all cases, we fixed $c=0.42$.

\section{$s$-wave: field scans at constant temperature}

\begin{tabular}{lccr}
\hline $\boldsymbol{T}(\mathrm{mK})$ & $\boldsymbol{\eta}$ & $\boldsymbol{\epsilon}$ & ${\text { RMSD }\left(\mathbf{1 0}^{-\mathbf{8}}\right)}$ \\
\hline 40 & $1.17(4)$ & $0.78(5)$ & 3.23 \\
130 & $1.01(2)$ & $0.74(4)$ & 1.83 \\
250 & $0.80(2)$ & $0.82(9)$ & 2.26 \\
350 & $0.75(4)$ & $1.0(1)$ & 1.24
\end{tabular}

$(s+s)$-wave: field scans at constant temperature

\begin{tabular}{lccr}
\hline $\boldsymbol{T}(\mathrm{mK})$ & $\boldsymbol{\eta}$ & $\boldsymbol{\epsilon}$ & ${\text { RMSD }\left(\mathbf{1 0}^{\mathbf{- 8}}\right)}$ \\
\hline 40 & $1.17(4)$ & $0.78(5)$ & 3.23 \\
130 & $1.08(2)$ & $0.74(4)$ & 1.87 \\
250 & $1.00(3)$ & $0.8(1)$ & 2.39 \\
350 & $1.01(6)$ & $1.1(1)$ & 1.34
\end{tabular}

$s$-wave: temperature scans at constant field

\begin{tabular}{lccc}
\hline $\boldsymbol{B}(\mathrm{T})$ & $\boldsymbol{\eta}$ & $\boldsymbol{\epsilon}$ & ${\text { RMSD }\left(\mathbf{1 0}^{-\mathbf{8}}\right)}$ \\
\hline 0.8 & $0.7(2)$ & $2(1)$ & 1.19 \\
1.6 & $1.5(5)$ & $1.8(5)$ & 2.72
\end{tabular}

$(s+s)$-wave: temperature scans at constant field

\begin{tabular}{lccr}
\hline $\boldsymbol{B}(\mathrm{T})$ & $\boldsymbol{\eta}$ & $\boldsymbol{\epsilon}$ & ${\text { RMSD }\left(\mathbf{1 0}^{-\mathbf{8}}\right)}$ \\
\hline 0.8 & $1.5(6)$ & $0.6(4)$ & 1.45 \\
1.6 & $1.10(3)$ & $0.8(2)$ & 2.11 \\
\hline \hline
\end{tabular}

tion from PPE close to $B_{\mathrm{c} 2}$, before entering the FFLO state, which then decreases the intensity abruptly.

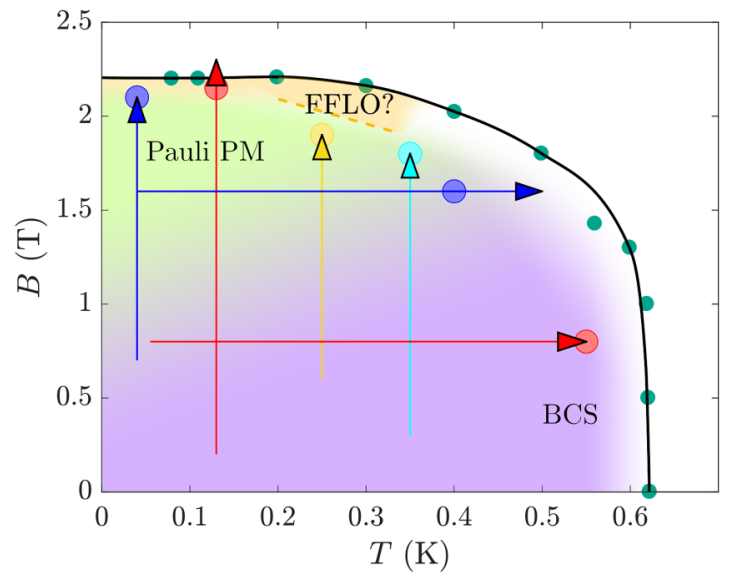

FIG. 5. $B-T$ phase diagram for superconductivity in $\mathrm{CeCu}_{2} \mathrm{Si}_{2}$ with $\mathbf{B} \| \mathbf{c}$. The different colors highlight the BCS region and where the Pauli paramagnetism is dominant at low temperatures and high fields and the FFLO region appearing close to the upper critical field at low temperatures. The dashed line indicates the limit of the FFLO region proposed by NMR studies [5]. Arrows show the field- and temperature scans covered by the SANS experiments. Shaded points show the highest field or temperature at which the vortex lattice was visible in each scan. The black line represents the upper critical field $B_{\mathrm{c} 2}$ as a function of temperature for $\mathbf{B} \| \mathbf{c}$ with the green-solid points representing experimental data obtained by ac susceptibility [5].
In our material, the flux lattice remains close to regular hexagonal for all fields [Fig. 2(b)] and no square to hexagonal transition is observed. This might be explained by a destabilisation of the square lattice due to Pauli paramagnetic effects [35], but more likely is the effect of an $s$-wave pairing as is discussed in Ref. [18]. In addition to these considerations, our measurements indicate a fully gapped $s$-wave model or preferably $s+s$ multigap behavior, which is also supported by heat capacity measurements $[10,11]$. For that reason we propose that $\mathrm{CeCu}_{2} \mathrm{Si}_{2}$ is a two-band $s$-wave superconductor.

In conclusion, we have performed small-angle neutron scattering measurements on the vortex lattice in $\mathrm{CeCu}_{2} \mathrm{Si}_{2}$ applying a magnetic field at various temperatures below $T_{c}$ and concluded that our results are consistent with two-band $s+s$-wave superconductivity. Pauli paramagnetic effects are clearly observed in the field dependence of the form factor, and we have proposed a model to fit these results. The decrease in the VL signal intensity immediately below $B_{\mathrm{c} 2}$ at low temperatures could be a further indication of the existence of the FFLO state in this heavy-fermion superconductor.

\section{METHODS}

The S-type sample used here is the same as that used in Ref. [2], with volume $6 \times 6 \times 8 \mathrm{~mm}^{3}$ and a total mass of $2.014 \mathrm{~g}$. It has a critical temperature of $T_{c} \approx 0.6 \mathrm{~K}$ and an upper critical field $B_{\mathrm{c} 2}(0) \approx 2.2 \mathrm{~T}$ [2]. Its low resistivity indicates a high-quality sample with electron mean free path longer than the superconducting coherence length (see Supplemental Material [24] for details). 
Our neutron measurements were carried out in several experiments at the D33 instrument of the Institut Laue-Langevin (ILL) [36,37], and the SANS-I instrument at the Swiss spallation neutron source SINQ, Paul Scherrer Institute (PSI). In the Supplemental Material [24], details are given of the individual experiments and the different data symbols used for results from each.

The vortex lattice was prepared by cooling the sample through $T_{c}$ to base temperature, while oscillating the field value by $\sim \pm 1 \%$ of the desired final value, which was held constant after cooling; this improves the perfection of the VL. After that, the sample and the cryomagnet could be rotated together about two perpendicular axes passing through the sample: $\omega$ about the vertical axis or $\phi$ about the horizontal axis perpendicular to the field. As the angle between the incoming neutron beam and the magnetic field was varied, the VL planes passed through the Bragg condition, giving a "rocking curve" of intensity of a diffraction spot, as shown in Fig. 1. Background data were measured with either $T>T_{c}$ or
$B>B_{\mathrm{c} 2}$ at the same rotation angles, and subtracted to give VL diffraction patterns, such as that shown in the inset of Fig. 1. Integrated intensities for the VL Bragg peaks were extracted from the rocking curve data using a Bayesian analysis procedure implemented in GRASP [38,39].

\section{ACKNOWLEDGMENTS}

We acknowledge helpful discussions with K. Machida and M. Ichioka about their first-principles calculations of the mixed state structure in Pauli-limited superconductors. This work was supported by the UK Engineering and Engineering and Physical Sciences Research Council (EPSRC), Grant No. EP/J016977/1. This work is based on experiments performed at the Institut Laue-Langevin (ILL), Grenoble, and the Swiss spallation neutron source SINQ, Paul Scherrer Institute, Villigen, Switzerland.
[1] F. Steglich, J. Aarts, C. D. Bredl, W. Lieke, D. Meschede, W. Franz, and H. Schäfer, Phys. Rev. Lett. 43, 1892 (1979).

[2] O. Stockert, J. Arndt, E. Faulhaber, C. Geibel, H. Jeevan, S. Kirchner, M. Loewenhaupt, K. Schmalzl, W. Schmidt, Q. Si et al., Nat. Phys. 7, 119 (2011).

[3] A. M. Clogston, Phys. Rev. Lett. 9, 266 (1962).

[4] K. Maki, Phys. Rev. 148, 362 (1966).

[5] S. Kitagawa, G. Nakamine, K. Ishida, H. S. Jeevan, C. Geibel, and F. Steglich, Phys. Rev. Lett. 121, 157004 (2018).

[6] A. I. Larkin and Y. N. Ovchinnikov, Zh. Eksp. Teor. Fiz. 47, 1136 (1964) [Sov. Phys. JETP 20, 762 (1965)].

[7] P. Fulde and R. A. Ferrell, Phys. Rev. 135, A550 (1964).

[8] N.-P. Ong and R. Bhatt, More Is Different (Princeton University Press, Princeton, 2001).

[9] H. A. Vieyra, N. Oeschler, S. Seiro, H. S. Jeevan, C. Geibel, D. Parker, and F. Steglich, Phys. Rev. Lett. 106, 207001 (2011).

[10] S. Kittaka, Y. Aoki, Y. Shimura, T. Sakakibara, S. Seiro, C. Geibel, F. Steglich, H. Ikeda, and K. Machida, Phys. Rev. Lett. 112, 067002 (2014).

[11] S. Kittaka, Y. Aoki, Y. Shimura, T. Sakakibara, S. Seiro, C. Geibel, F. Steglich, Y. Tsutsumi, H. Ikeda, and K. Machida, Phys. Rev. B 94, 054514 (2016).

[12] G. Pang, M. Smidman, J. Zhang, L. Jiao, Z. Weng, E. M. Nica, Y. Chen, W. Jiang, Y. Zhang, W. Xie et al., Proc. Natl. Acad. Sci. U.S.A. 115, 5343 (2018).

[13] Y. Li, M. Liu, Z. Fu, X. Chen, F. Yang, and Y.-f. Yang, Phys. Rev. Lett. 120, 217001 (2018).

[14] A. D. Bianchi, M. Kenzelmann, L. DeBeer-Schmitt, J. S. White, E. M. Forgan, J. Mesot, M. Zolliker, J. Kohlbrecher, R. Movshovich, E. D. Bauer et al., Science 319, 177 (2008).

[15] M. Ichioka and K. Machida, Phys. Rev. B 76, 064502 (2007).

[16] L. DeBeer-Schmitt, M. R. Eskildsen, M. Ichioka, K. Machida, N. Jenkins, C. D. Dewhurst, A. B. Abrahamsen, S. L. Bud'ko, and P. Canfield, Phys. Rev. Lett. 99, 167001 (2007).

[17] A. S. Cameron, J. S. White, A. T. Holmes, E. Blackburn, E. M. Forgan, R. Riyat, T. Loew, C. D. Dewhurst, and A. Erb, Phys. Rev. B 90, 054502 (2014).
[18] M. Enayat, Z. Sun, A. Maldonado, H. Suderow, S. Seiro, C. Geibel, S. Wirth, F. Steglich, and P. Wahl, Phys. Rev. B 93, 045123 (2016).

[19] S. L. Thiemann, Z. Radović, and V. G. Kogan, Phys. Rev. B 39 , 11406 (1989).

[20] V. G. Kogan, M. Bullock, B. Harmon, P. Miranović, Lj. Dobrosavljević-Grujić, P. L. Gammel, and D. J. Bishop, Phys. Rev. B 55, R8693 (1997).

[21] V. G. Kogan and J. R. Clem, Phys. Rev. B 24, 2497 (1981).

[22] J. S. White, P. Das, M. R. Eskildsen, L. DeBeer-Schmitt, E. M. Forgan, A. D. Bianchi, M. Kenzelmann, M. Zolliker, S. Gerber, J. L. Gavilano et al., New J. Phys. 12, 023026 (2010).

[23] D. K. Christen, F. Tasset, S. Spooner, and H. A. Mook, Phys. Rev. B 15, 4506 (1977).

[24] See Supplemental Material at http://link.aps.org/supplemental/ 10.1103/PhysRevB.104.184508 for (i) models for the form factor with orbital currents only, (ii) for the estimation of electron mean free path in our sample, and (iii) further details of the different experiments performed.

[25] M. Ichioka (private communication).

[26] E. Brandt, Phys. Status Solidi B 51, 345 (1972).

[27] J. S. White, R. W. Heslop, A. T. Holmes, E. M. Forgan, V. Hinkov, N. Egetenmeyer, J. L. Gavilano, M. Laver, C. D. Dewhurst, R. Cubitt, and A. Erb, Phys. Rev. B 84, 104519 (2011).

[28] L. Kramer and W. Pesch, Z. Phys. 269, 59 (1974).

[29] K. Yosida, Phys. Rev. 110, 769 (1958).

[30] R. Prozorov and R. W. Giannetta, Supercond. Sci. Technol. 19, R41 (2006).

[31] K. M. Suzuki, Y. Tsutsumi, N. Nakai, M. Ichioka, and K. Machida, J. Phys. Soc. Jpn. 80, 123706 (2011).

[32] K. M. Suzuki, K. Machida, Y. Tsutsumi, and M. Ichioka, Phys. Rev. B 101, 214516 (2020).

[33] T. Yamashita, T. Takenaka, Y. Tokiwa, J. A. Wilcox, Y. Mizukami, D. Terazawa, Y. Kasahara, S. Kittaka, T. Sakakibara, M. Konczykowski et al., Sci. Adv. 3, e1601667 (2017). 
[34] P. Gegenwart, C. Langhammer, C. Geibel, R. Helfrich, M. Lang, G. Sparn, F. Steglich, R. Horn, L. Donnevert, A. Link, and W. Assmus, Phys. Rev. Lett. 81, 1501 (1998).

[35] N. Hiasa and R. Ikeda, Phys. Rev. Lett. 101, 027001 (2008).

[36] E. Blackburn, E. Campillo, R. Cubitt, E. M. Forgan, A. T. Holmes, Z. Huesges, E. Jellyman, R. Riyat and O. Stockert (2018), Vortex lattice of the heavy fermion superconductor $\mathrm{CeCu} 2 \mathrm{Si} 2$. Institut Laue-Langevin (ILL); doi:10.5291/ILLDATA.5-42-463.
[37] E. Blackburn, R. Cubitt, M. R. Eskildsen, E. M. Forgan, H. Kawano-Furukawa, A. T. Holmes, E. Jellyman, S. Kuhn, L. Lemberger, R. Riyat and M. Takahashi (2016), Pauli limited behaviour in an iron-based superconductor? Institut LaueLangevin (ILL); doi:10.5291/ILL-DATA.5-42-376.

[38] Graphical Reduction and Analysis SANS Program for MATLAB $^{\mathrm{TM}}$, https://www.ill.eu/users/support-labsinfrastructure/software-scientific-tools/grasp.

[39] A. T. Holmes, Phys. Rev. B 90, 024514 (2014). 